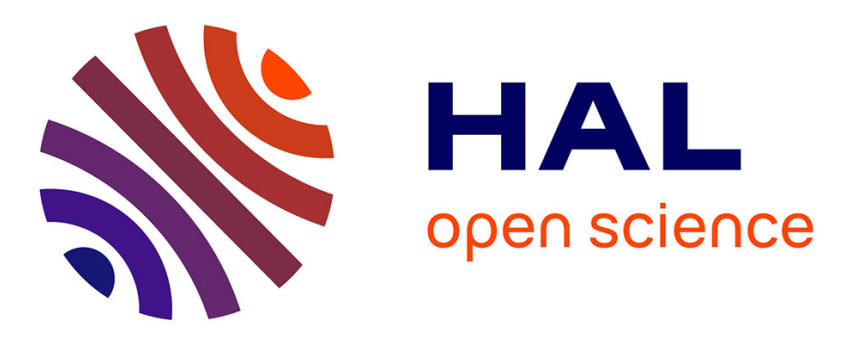

\title{
Drying kinetics driven by the shape of the air/water interface in a capillary channel
}

Emmanuel Keita, Stephan A Koehler, Pamela Françoise Faure, David A. Weitz, Philippe Coussot

\section{- To cite this version:}

Emmanuel Keita, Stephan A Koehler, Pamela Françoise Faure, David A. Weitz, Philippe Coussot. Drying kinetics driven by the shape of the air/water interface in a capillary channel. European Physical Journal E: Soft matter and biological physics, 2016, 39 (2), 10.1140/epje/i2016-16023-8 . hal-02136419

\section{HAL Id: hal-02136419 https://hal-enpc.archives-ouvertes.fr/hal-02136419}

Submitted on 22 May 2019

HAL is a multi-disciplinary open access archive for the deposit and dissemination of scientific research documents, whether they are published or not. The documents may come from teaching and research institutions in France or abroad, or from public or private research centers.
L'archive ouverte pluridisciplinaire HAL, est destinée au dépôt et à la diffusion de documents scientifiques de niveau recherche, publiés ou non, émanant des établissements d'enseignement et de recherche français ou étrangers, des laboratoires publics ou privés. 


\title{
Drying kinetics driven by the shape of the air/water interface in a capillary channel ${ }^{\star}$
}

\author{
Emmanuel Keita ${ }^{1,2, a}$, Stephan A. Koehler ${ }^{2}$, Paméla Faure ${ }^{1}$, David A. Weitz ${ }^{2}$, and Philippe Coussot ${ }^{1}$ \\ 1 Laboratoire Navier, Université Paris-Est, France \\ 2 School of Engineering and Applied Sciences and Physics Department, Harvard University, USA
}

Received 3 September 2015 and Received in final form 12 November 2015

(c) EDP Sciences / Società Italiana di Fisica / Springer-Verlag 2016

\begin{abstract}
We look at the drying process in a simple glass channel with dominant capillary effects as is the case in microfluidics. We find drying kinetics commonly observed for confined geometry, namely a constant period followed by a falling rate period. From visualization of the air/water interface with high resolution, we observe that the drying rate decreases without a drying front progression although this is the usually accepted mechanism for confined geometries. We show with FEM that in our specific geometry the falling rate period is due to changes in the shape of the air-water interface at the free surface where most evaporation occurs. Our simulations show that the sensitivity of the drying rate to the shape of the first air-water interface from the sample free surface implies that slight changes of the wetting or pinning conditions can significantly modify the drying rate.
\end{abstract}

\section{Introduction}

Drying of channels is of great interest for microfluidics process where drying induces passive flow [1], cools down devices [2], concentrates suspension [3,4] and leads to controlled crystal growth [5]. Besides capillary channels are also considered as model porous media in which it is possible to observe the details of the air-water interface in time. Studying drying kinetics from such simple configurations makes it possible to understand the basics mechanisms involved in evaporation from a confined geometry.

So far studies on 2D porous systems mainly focused on air/liquid interfaces throughout the sample (InvasionPercolation process) [6-8]. Detailed observations were possible in unique long and slender channels that are closed at one end and exposed to the atmosphere at the other end. It was shown that evaporation from a cylindrical channel decreases with $1 / \sqrt{t}$ as water vapor diffuses over an increasing length from the meniscus to the channel end [9]. But changing the channel cross sectional shape strongly influences the water distribution. In particular the drying rate from channels with corners remains constant and enhanced relatively to vapor diffusion because liquid films in the corners extend to the free surface and sources the water for evaporation [10-12].

* Contribution to the Topical Issue "Wetting and Drying: Physics and pattern Formation", edited by Duyang Zang, Ludovic Pauchard and Wei Shen.

a e-mail: emmanuel.keita@ifsttar.fr
In $[10,11,13]$ the subsequent decrease of the drying rate was associated with a depinning of the liquid films from the free surface resulting from gravitational and viscous effects. The high wettability of the solid by the fluid is necessary for water to stay in channel edges as water wets more surfaces. If the contact angle is too high, films are geometrically impossible in a right angle corner $[10,14]$; in this situation drying is a slow diffusive process as in cylindrical tube. Therefore water distribution is very sensitive to wetting or pinning conditions and can modify the drying kinetics.

Drying from 3D porous media exhibits some similarity with drying from simple channels $[13,15,11,16]$, but it is much more difficult to relate the exact shape of the air-liquid interface to the drying rate. Sophisticated 3D imaging tools such as MRI indicate that when a dry front develops the drying rate decreases; but the resolution is not sufficient to determine the distribution of water within individual pores [17-19]. Other studies investigated the wetting of porous media [20-22] without providing much insight into the impact of the exact shape of the liquid-air interface on the drying rate.

It is useful to recall the drying principles of hydrophilic confined geometries [13,23-26]. Regardless of the complexity of the material structure (from square capillary to soils) two main stages of drying are observed for an initially water-saturated device. The basic physical origin of these regimes is as follows:

i) Drying rate starts with a Constant Rate Period (CRP), where the drying rate remains constant until the 
majority of water is evaporated. Here the drying rate is set by external conditions such as air flow, temperature and humidity. It has been shown that for this regime the capillary pressure is sufficient to drive water to the sample outer surface $[23,24]$.

ii) This is followed by the Falling Rate Period (FRP) where the drying rate decreases and approaches zero. The proposed mechanism for this decrease is that below a certain saturation value (ratio of water to total volume), viscous dissipation is too high for capillary pressure to source a flow to the outer surface equaling the CRP drying rate. Consequently a drying front invades the medium causing evaporation to increasingly slow down because water vapor has to diffuse from the receding front to the free surface.

Here we study the drying of a single channel having a rectangular cross-section that is held horizontal to remove gravitational effects. We combine high-resolution weighing with microscopy to accurately record the drying process. Similar to porous media, we observe that evaporation rate has two regimes: CRP followed by a FRP. These observations lead us to carry out FEM simulations which provide an insight in the origin of these trends. We thus show how capillarity sets the water distribution and the decrease in evaporation rate in our specific geometry is due to variations in the shape of the liquid-air interface around the entrance of the channel.

\section{Materials and methods}

\subsection{Glass capillary}

We used a borosilicate glass channel with a high-aspect ratio rectangular cross-section $(W=2 \mathrm{~mm} ; H=0.1 \mathrm{~mm}$, $L=10 \mathrm{~mm}$ from Vitrocom $\left.{ }^{\circledR}\right)$. It is important to note that the channel cross section is not perfectly rectangular (see fig. 1b). The glass is cleaned with Isopropanol, and the contact angle is small, between $5^{\circ}$ and $10^{\circ}$. Accordingly when the channel is in contact with deionized water it is filled due to capillary suction. Once filled with water, one of the channel ends is closed off with epoxy glue such that evaporation only occurs at the other end.

\subsection{Drying conditions and measurements}

We tracked the drying process of the filled horizontal glass channel using two separate measurements, which are imaging and weighing. For both experiments the temperature was held at $25^{\circ} \mathrm{C}$ and the relative humidity at $50 \%$ by flowing air at a rate of $10 \mathrm{~mL} / \mathrm{min}$ across the channel opening. The rate of evaporation was recorded by taking the mass every 25 seconds using a TGA (thermogravimetric analysis from Texas Instruments). We obtain the mean saturation $\phi=V_{\text {water }} / V_{\text {water ini from the ratio of the water }}$ current mass to its initial mass. The noise is of the order of $0.2 \mu \mathrm{g}$ and the initial water mass $m_{\text {water ini }} \approx 1 \mathrm{mg}$, therefore the saturation is obtained with an accuracy of
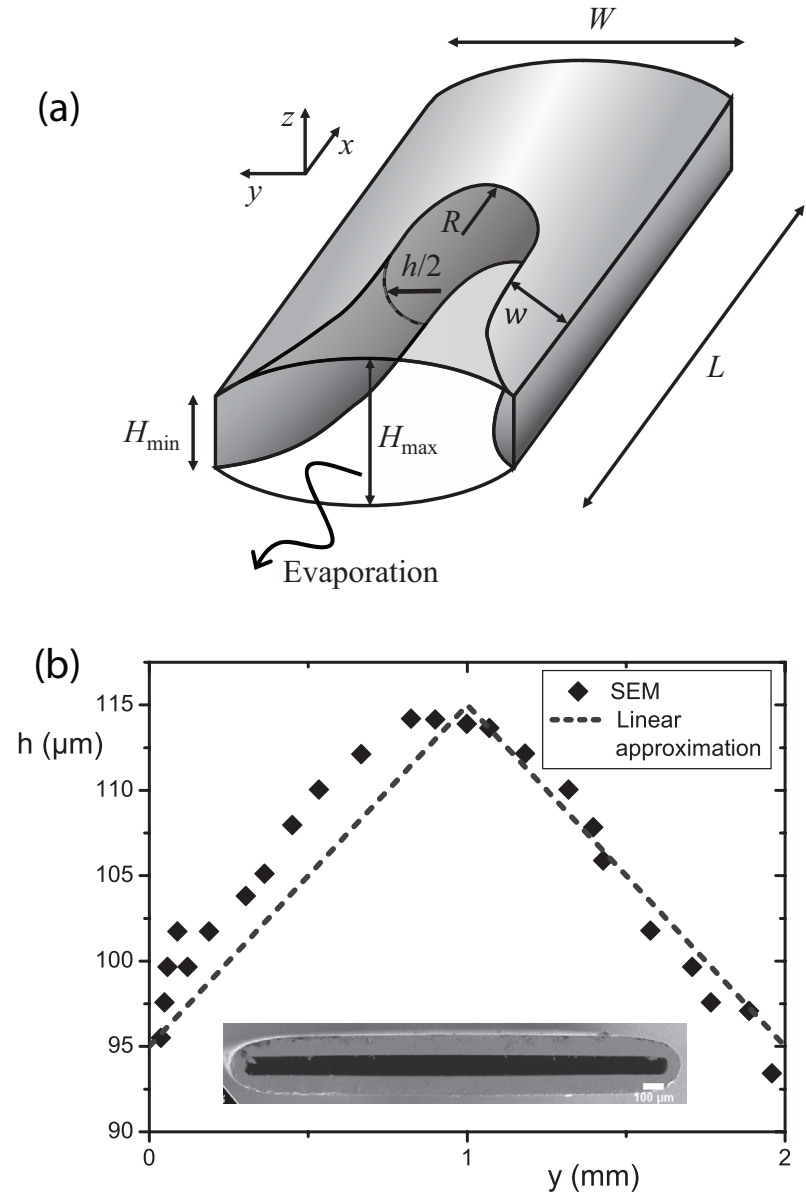

Fig. 1. Schematic of the experimental geometry: a glass channel with rectangular-like cross section closed at one end and at the entrance open for evporation. The receeding air/water interface is qualitatively sketched. (b) Channel height, $h$, measured from the SEM images of our geometry a function of the transverse position, $y$. The linear approximation is used for modeling (dash line).

$\phi \approx 2 \cdot 10^{-4}$. Here we exemplify the repeatability and the uncertainty through a comparison between the results on two samples. In a separate experiment we imaged the channel every 10 seconds with a microscope in transmission mode. We measure $\phi$ from the position of the air/water interfaces as observed from above which neglects the out-of-plane curvature. Due to this approximation, lensing effects of the interface and limited pixel resolution $(4.3 \mu \mathrm{m})$, the optical values of the saturation are significantly less accurate than those obtained from weighing.

\subsection{Finite element modeling}

To understand the drying kinetics, we model the local evaporation from the air/water interface by calculating the vapor density field, $n$, implementing 2D FEM using MATLAB. We use the function "adaptmesh" to generate the mesh and refine triangles with high vapor gradients. 


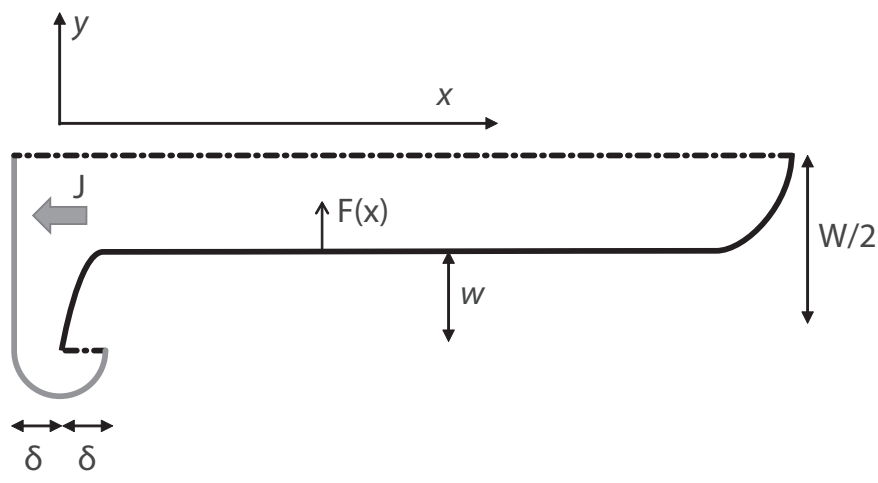

Fig. 2. Schematic of the domain used for 2D FEM modeling. The air-water interface is shown as a solid black curve, and the dashed lines indicate no-flux boundary conditions. The humidity is fixed at $50 \%$ for the gray curve, which is a distance delta away from the channel. $F$ is the local evaporation from the interface and the drying rate $J$ is the flux of water vapor through the channel entrance.

The 2D geometry is justified by the large aspect ratio of in-plane geometric features compared to the much smaller vertical out-of-plane features. We neglect the slight tapering of the channel rectangular cross-section. For the simulations the shape of the interface is externally set; we either use microscopic images or simplified idealizations. 3D simulations may have given a more realistic representation of the air/water interface shape and of vapor diffusion outside the channel. It seems relevant to keep the $2 \mathrm{D}$ for a first distinction of the main phenomena.

We assume a quasi-static air-water interface, indeed the vapor diffusion velocity is of the order of $D / L \approx$ $10^{-3} \mathrm{~m} \mathrm{~s}^{-1}$, where water vapor diffusion coefficient is $D=$ $2.7 \cdot 10^{-5} \mathrm{~m}^{2} \mathrm{~s}^{-1}$, and far exceeds the experimentally observed drying velocity $V_{\text {drying }} \approx 2 \cdot 10^{-6} \mathrm{~m} \mathrm{~s}^{-1}$ (see eq. (2)). As a consequence the Laplace equation $(\Delta n=0)$ captures the spatial field of vapor density. Along the liquid-air interface the vapor density is maximal, $n=n_{0}=23.4 \mathrm{~g} \mathrm{~m}^{-3}$, which is indicated by the solid black curve in fig. 2 . The external air flow produces a vapor density of $n=n_{0} / 2$ at a distance $\delta$ from the channel entrance, shown as a gray curve in fig. 2. $\delta$ represents the influence of the external conditions during the CRP and does not vary during the experiment. Along the centerline axis and glass wall of the capillary we use a no-flux boundary condition, $\nabla n \cdot s=0$ where $s$ is the normal vector, which is shown as dashed lines in fig. 2. We solve for the local evaporation flux along the air/water interface: $F=-D \cdot \nabla n$. The drying rate is the integral along the channel entrance, $J=$ $-\int_{x=0} D \cdot \nabla n \cdot \mathrm{d} s$.

\section{Results}

\subsection{Imaging}

Initially the channel is completely filled with water (saturation $\phi=1$ ) and over time the air/water interface curves inwards due to water loss from evaporation, while water remains pinned at both edges of the entrance (see fig. 3a). As drying proceeds a finger invades the channel (see the schematic in fig. 1 and fig. 3b-d). The main meniscus keeps a constant shape (approximately a half-circle of radius $r_{\mathrm{c}}=0.6 \mathrm{~mm}$ ) and progresses with a constant speed towards the end of the channel, leaving behind two water layers of constant thickness $w=0.4 \mathrm{~mm}$ along the channel edges (see fig. 3c-d). Behind the fingertip air occupies $60 \%$ of the cross-section. Accordingly, once the saturation has dropped to 0.4 the fingertip has reached the channel end the two water layers begin to thin uniformly (see fig. 3e-f). As $w$ becomes of the order of the channel height, $H=100 \mu \mathrm{m}$, the films are difficult to discern, as shown in fig. $3 \mathrm{~g}$

For each position along channel axis, we measure the distance between the interface and the channel edge as the shortest distance separating the two black lines on the upper half of images of fig. 3. In the drying process we observe three characteristic profile shapes which evolve in a self-similar fashion. Starting from the back of the channel, these are: the main meniscus, the thin films at the sides and the entrance profile. The main meniscus has a characteristic curved profile, resembling a finger, which advances into the channel (see fig. 3b-d). As the finger advances, the side films increase in length while maintaining their width $w \approx 0.38 \mathrm{~mm}$ and film thickness is constant for $x>0.4 \mathrm{~mm}$ as shown for the times $17-103 \mathrm{~min}$ in fig. $4 \mathrm{a}$. As the water films shrink, the entrance profile curves further into the channel and constant thickness is established sooner (at $x \approx 0.1 \mathrm{~mm}$ for $163 \mathrm{~min}$, see fig. $4 \mathrm{~b}$ ). Moreover at 163 min the interface has receded by tenths of microns from the entrance of the tube.

\subsection{Drying rates}

The mass measurements are sufficiently accurate to allow differentiation for obtaining the evaporation rate, which is shown as a function of saturation in fig. 5. However the derivation procedure tends to increase the impact of data fluctuations. This explains that the final uncertainty on drying rate is of the order of $10 \%$ (see an example of two results for the same experiment in fig. 5). Using image analysis to determine the water volume we also obtain the evaporation for shorter times, which is shown as gray curve in fig. 5. The agreement between these two types of measurement is good over the range $\phi=0.5$ to 0.9 . Accurate measurements at $\phi=1$ are difficult, but initially it is clear that the drying rate decreases by more than $30 \%$ between $\phi=1$ and 0.9 . The majority of the water evaporates at a constant drying rate, defining the CRP for $\phi=0.3$ to 0.9 . For lower water saturations, $\phi<0.3$ the evaporation rate decreases to zero. The drying rate starts to decrease around $\phi=0.3-0.25$ then there is a sharp decrease in the range $\phi=0.2-0.1$.

\subsection{FEM using experimentally observed interfaces}

We implement the 2D FEM scheme for the interfaces extracted from the channel images (see fig. 3) and obtain the 
(a)

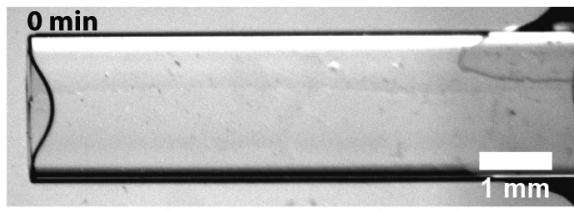

(b)

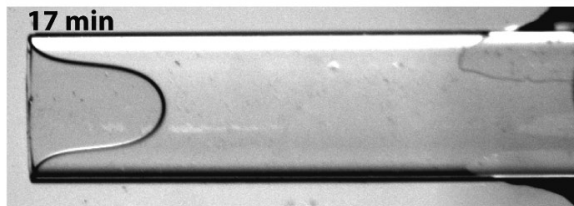

(c)

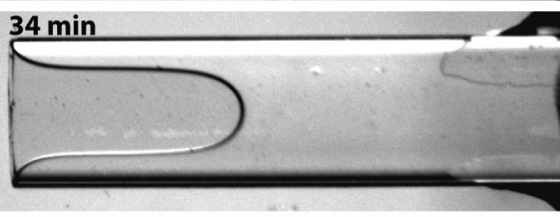

(d)

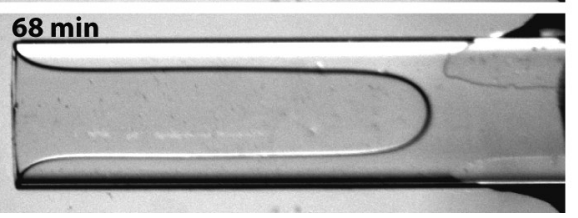

(e)

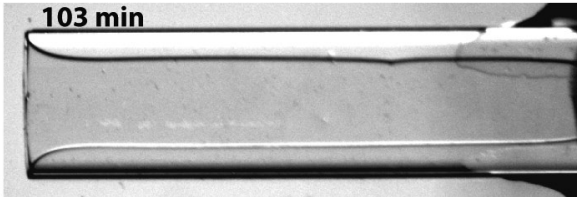

(f)

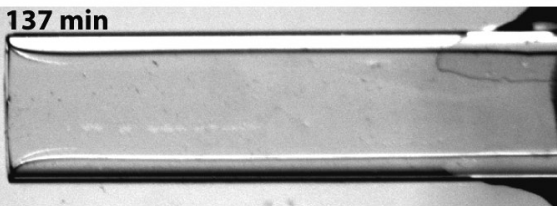

(g)

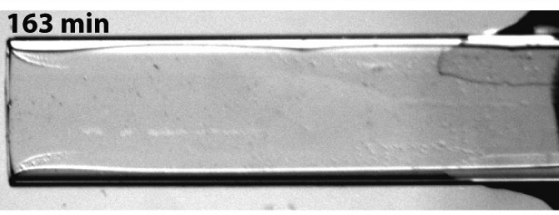

Fig. 3. Top views of the channel (open on the left) at different times during drying: (a) initial and after 17 (b), 34 (c), 68 (d), 103 (e), 137 (f) and $163 \mathrm{~min}(\mathrm{~g})$. The scale bar denotes $1 \mathrm{~mm}$ and applies to all panels.

(a)

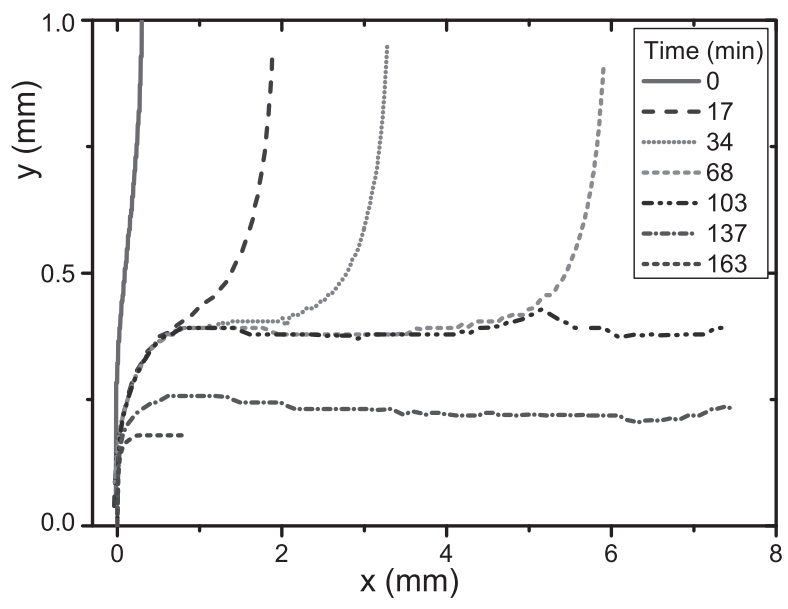

(b)

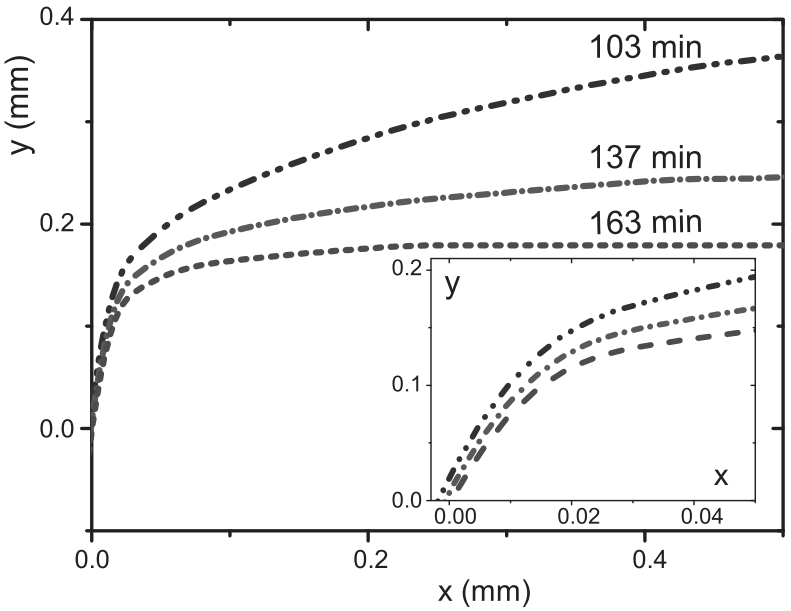

Fig. 4. (a) Shape of the air/water interface for images from fig. 3, drying times are indicated in minutes. We present here the upper half of the images of the interface. The channel is open at $x=0$ and its axis is $y=1 \mathrm{~mm}$. (b) Interface close to the entrance of the channel at the end of the drying. The inset is a zoom on the first $50 \mu \mathrm{m}$ of the channel entrance for the same interfaces.

field of relative humidity in channel air space (see fig. 6). At the beginning, the interface is almost straight at the free surface (fig. 6a) and the vapor gradient is uniform. As the air finger enters (fig. 6b), the air close to the main meniscus is saturated with water vapor. When the main meniscus progresses, the air is saturated with water vapor for $x>1.5-2 \mathrm{~mm}$, the liquid water is at equilibrium with the air (fig. 6c). Close to the entrance, the gradient of vapor density is high and so is the local evaporation.

The drying rate computed from the 2D FEM, $J$, remains constant as the main meniscus recedes into the channel and decreases as the films shrink. The modeled drying rate is in good agreement with the weighing and optical measurements, as shown by the gray squares in fig. 5, and we thus have established their utility for gaining further insight into the drying kinetics.

\section{Analysis of the different drying regimes}

We perform a comprehensive analysis of the drying process based upon image analysis and TGA measurements coupled with 2D FEM simulations to study the physical origin of the successive evaporation regimes. 


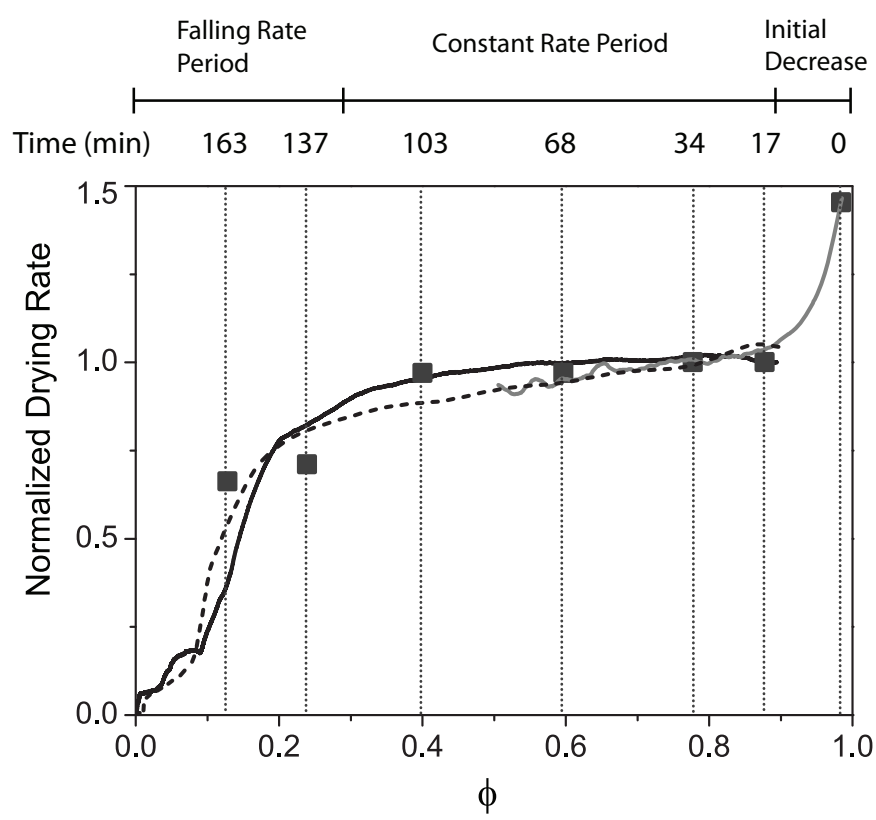

Fig. 5. Drying rate, normalized at CRP, as a function of the saturation, from two measurements by TGA (black continuous and dotted curves) and from image analysis (gray curve). Times refer to pictures of fig. 3 . The gray squares are the drying rate computed from FEM.

\subsection{Initial decrease}

Initially the channel is completely filled and the air-water interface is flat. Consequently the entire interface is the closest to the dry air surrounding the channel, thereby resulting in the greatest drying rate. Assuming there is no air flow in the boundary layer of thickness $\delta$, the rate of evaporation is proportional to the gradient of vapor density according to Fick's law:

$$
\frac{\mathrm{d} m}{\mathrm{~d} t}{ }_{t=0} \sim \frac{D n_{0} W H}{2 \delta} .
$$

However we do not have reliable data concerning the drying rate in the very first times of a test. In order to determine the value of $\delta$ we compare the drying rate drying rate $J_{\mathrm{CRP}}$ predicted from simulations during the CRP (i.e. at $68 \mathrm{~min}$, see below), using the observed shape of the interface at that time, to the drying rate measured in that regime. We find $\delta=5 \cdot 10^{-5} \mathrm{~m}$, and we deduce from (1) that the drying rate at the very beginning of the test, i.e. when the interface is straight, should be equal to 3.5 times the CRP drying rate. The validity of this approach is supported by the good agreement between the drying rate computed by FEM with the first reliable measurement (see fig. 5).

Between the beginning of the test and the CRP the drying rate decreases as the air finger forms and water vapor diffuses over an increasing length (see gray line in fig. 5). Over time the interface becomes increasingly concave, as shown in fig. 3. Since the evaporation flux decreases with distance from the dry air the drying rate rapidly drops off as the interface starts to become concave: it is already close to the CRP drying rate after a small decrease of saturation.

\subsection{Constant rate period}

The Constant rate period (CRP) starts at about 15 minutes and extends from a saturation of 0.9 down to around 0.25 , making it the dominant feature of the drying rate curve shown in fig. 5. The air finger is established roughly when its length is comparable to its width. Thus we estimate that the transition to $\mathrm{CRP}$ occurs at a saturation around $\phi=1-\frac{(W-2 w)^{2}}{W L}$. In our geometry, we obtain $\phi=0.9$ in very good agreement with observations. Moreover if the channel length is increased, this transition will occur at a saturation closer to 1.

During this regime a growing finger penetrates deeper into the channel while preserving the interfacial shape at the entrance of the channel (see fig. 3). Capillarity pumps water from the reservoir behind the finger to the entrance where evaporation is primarily occurring $[23,15,16,10]$. Below we first demonstrate that in CRP viscous dissipation has a negligible effect on drying, thus capillarity set the water distribution. Then we show that a constant curvature sets the shape of the air/water interface.

The pressure drop due to viscous flow through the side films depends on the mean velocity of the flowing water, which can be approximated using

$$
V_{\text {drying }}=(1 / w H \rho) \mathrm{d} m / \mathrm{d} t \approx 2 \cdot 10^{-6} \mathrm{~ms}^{-1},
$$

where $w$ is the width and $H$ the height (see fig. 8a-b). Accordingly the viscous pressure drop along the side film is of the order of

$$
\Delta p_{\text {vis }} \sim \frac{\mu L}{H w} V_{\text {drying }} \sim 1 \mathrm{mPa},
$$

where $\mu$ is the viscosity. This pressure drop causes a change in the interfacial curvature of the film. Because the wetting angle is close to zero, and the in-plane curvature is zero, the scale of the pressure as given by Laplace's law is $p_{c} \sim \frac{\gamma}{H} \sim 1 \mathrm{kPa}$ where $\gamma$ is the surface tension. Therefore the Laplace pressure is order of magnitude larger than the stress associated with viscous flow. Exact computations of the flow [14,27] suggest that the viscous pressure is 100 times higher than in eq. (3) but still much smaller than the capillary pressure. Meter long channels are required for viscous dissipation to be of the same order as capillary pressure. Thus changes in curvature due to viscous flow are negligible compared to the mean curvature. Consequently in this regime the air/water interface has a constant curvature. Thus the water distribution is at equilibrium and set by the capillarity.

The interface observed in CRP has two dominant features, which are the circular concave fingertip and the two side layers. Over time the fingertip moves towards the end of the channel while preserving its shape, and the two side layers maintain their thickness, as shown in fig. 4. The interface shape is set by the channel cross-sectional shape, 


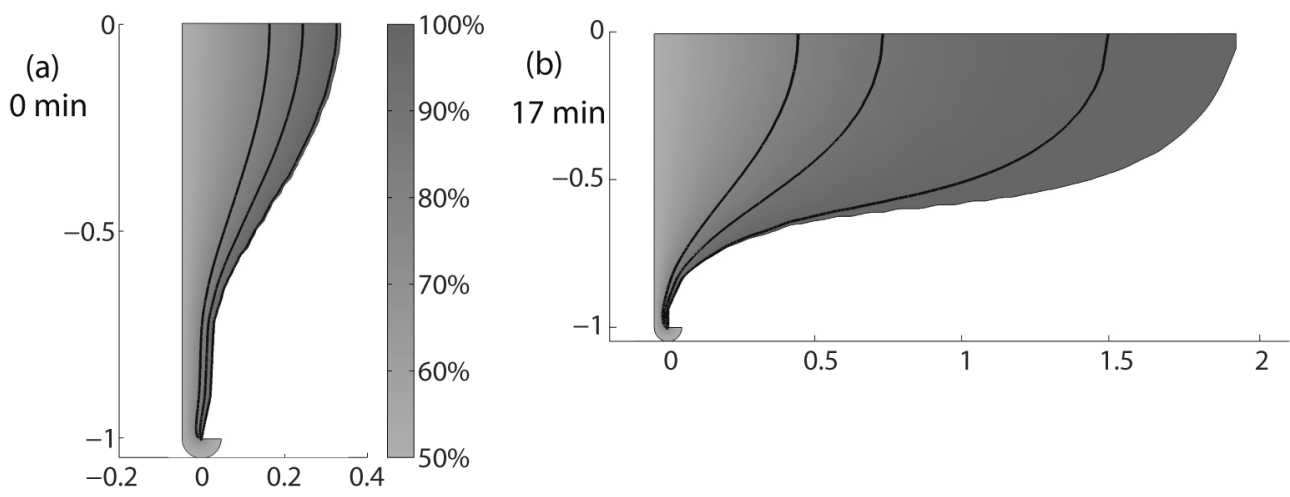

(c)

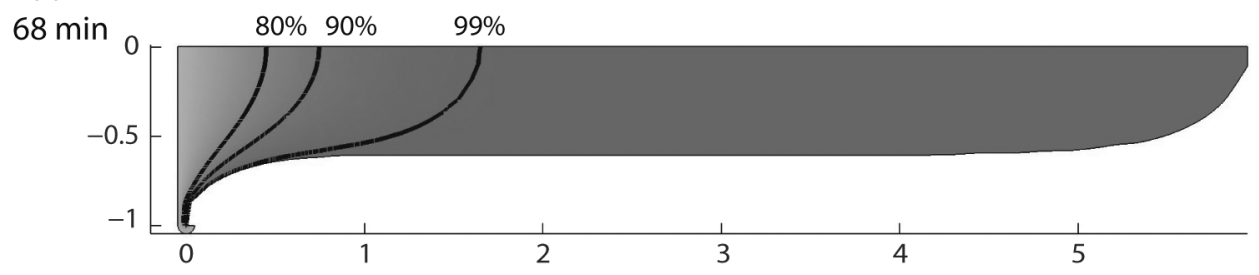

Fig. 6. Fields of relative humidity $n / n_{0}$ for the observed air/water interfaces as shown in fig. 3 (axes scale is millimeter). The air is saturated with water at the air/water interface and $n / n_{0}=50 \%$ at a distance $\delta$ from the channel entrance (light gray); as shown in fig. 2. The gray scale is common to all figures. The black lines indicate curves of iso-humidity ( 80,90 or $99 \%$ ).

the small wetting angle and a constant mean curvature as shown in fig. $7 \mathrm{a}-\mathrm{b}$.

The water distribution is different from the one observed in a square cross-section channel where four water films are in each of the corners $[13,11]$. Since in CRP the curvature is constant we can describe the shape of the interface by taking into account channel geometry [28]. Thus the sum of the in-plane curvature and the out-ofplane curvature $\frac{1}{r_{x y}}+\frac{1}{r_{y z}}$ is constant because due to the small wetting angle the second radius is half the channel height, $r_{y z} \approx \frac{h}{2}$.

As illustrated in fig. 1a and measured with SEM, the spacing is larger $\left(H_{\max }=115 \mu \mathrm{m}\right)$ in the center and narrower $\left(H_{\min }=95 \mu \mathrm{m}\right)$ at the edges. We consider that the variation is linear from the edge to the center, as shown in fig. 1b. We can calculate the curvature in some specific points of the air/water interface. At the main meniscus the total curvature is approximately $2 / H_{\max }+1 / r_{\mathrm{c}} \approx 19 \mathrm{~mm}^{-1}$. The straight portion of interface is $w=0.4 \mathrm{~mm}$ away from the edge, therefore the vertical spacing is $h=105 \mu \mathrm{m}$ (see fig. $1 \mathrm{~b}$ ) and the total curvature $2 / h \approx 19 \mathrm{~mm}^{-1}$. Finally, at the entrance of the channel, using $w$ as radius of curvature (but in a convex shape) in the plane of observation and the minimum spacing (along the edge) as the second curvature, we find $2 / H_{\min }-1 / w \approx 18.5 \mathrm{~mm}^{-1}$. In agreement with low viscous dissipation (eqs. (2) and (3)), the total curvature is uniform along the interface and capillary equilibrium is maintained everywhere.

More generally capillary equilibrium and geometry set the existence of two thick films rather than four corners films. The radius of curvature $r$ varies linearly with the distance from the edge as $2 r=h=a(w+m)+H_{\min }$, where $a$ is the slope of the spacing. Decreasing $w$ also decreases the radius of curvature $r$ as shown by the circular arc (gray dotted), simultaneously $r_{\mathrm{c}}$ increases. The geometrical constrains of fig. 7 link the curvature at the main meniscus and the thickness $w$. Moreover the curvature at the main meniscus (left term) and along the films (right term) are equal

$$
\frac{1}{r_{\mathrm{c}}(w)}+\frac{2}{H_{\max }}=\frac{2}{h(w)} .
$$

$w$ is then the solution of a quadratic equation and depends on the slope $a$ and the aspect ratio $\frac{W}{H_{\min }}$ (see fig. 7c). A given geometry is characterized by $\left(a, \frac{W}{H_{\min }}\right)$ which accept a unique films thickness $w$ setting a constant curvature at the main meniscus and the films. A negative value for $w$ means that water is only present in the 4 corners where air/water interface can adjust its curvature (see fig. $7 \mathrm{~b}$ ). In our geometry, $\frac{W}{H_{\min }}=21$ and $a=2 \cdot 10^{-2}$, we obtain $w=0.4 \mathrm{~mm}$ in very good agreement with our observations (see fig. 4). The same slope $a$ with $\frac{W}{H_{\min }}=10$ would lead to $w<0$. As $a$ is close to 0 , the spacing is constant and films curvature is constant with thickness thus $w<0$. As the section gets closer to a square, $\frac{W}{H_{\min }}$ approaches 1 , there are no values for $a$ where $w>0$ and the water is always in 4 corners films in agreement with previous observations [10]. For high aspect ratio, the films are very likely, indeed the smallest $a$ such as $w>0$ scales with $\frac{H_{\min }}{W}$.

To gain further insight into the evaporation process, we perform numerical simulations where the evaporation flux is calculated for a given shape. We implement the 2D FEM scheme for the interfaces extracted from the channel images of air/water interface and obtain the field of 
(a)

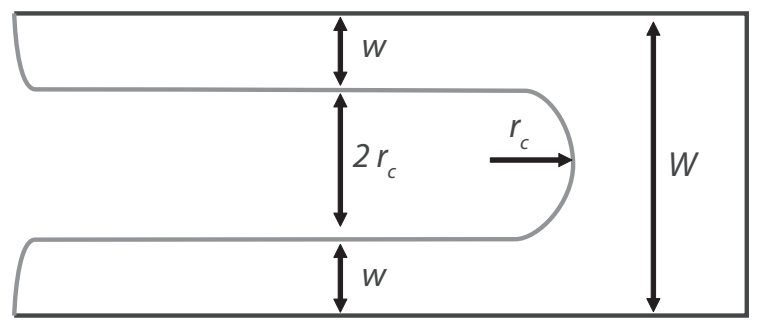

(b)

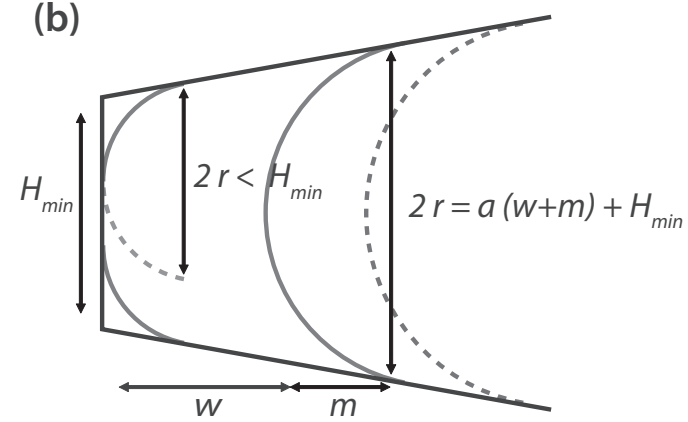

(c)

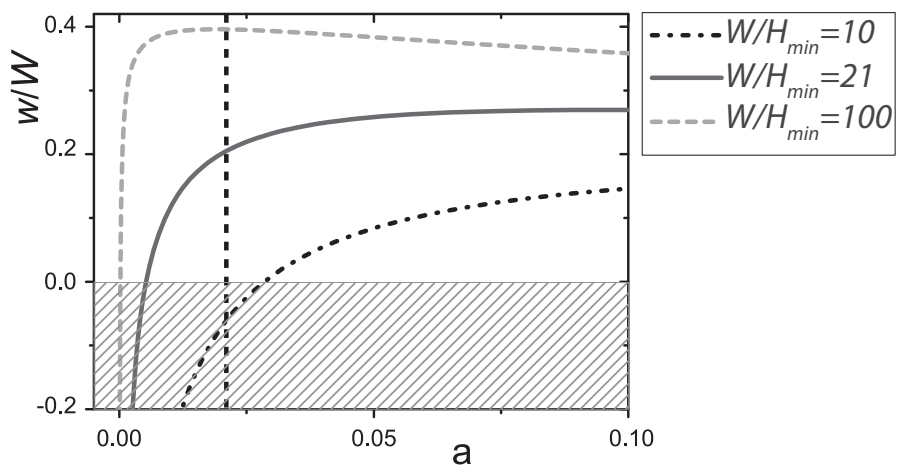

Fig. 7. (a) Schematic of the channel in the plane of observation. The width $W$ constrains the water film width $w<W / 2$, and the in-plane radius of in-plane curvature of the main meniscus $r_{\mathrm{c}}<\frac{W}{2}-w$. (b) Side view of the channel showing the interface with two side layers $(w>0)$ and four corner layers $(w<0)$. The channel spacing has a slope $a$, and the in-plane projected width of the meniscus is $m$. (c) Thickness of the water layer $w$ as a function of the slope $a$ calculated from eq. (4) setting a constant curvature for different aspect ratios of the channel $\frac{W}{H_{\min }}$. The solid curve shows our geometry where $\frac{W}{H_{\min }}=21$ and the dashed vertical line shows the value of our slope, $a=2 \cdot 10^{-2}$. The values of $w<0$ correspond to situation where the layers are in the 4 corners as depicted on (b).

relative humidity in channel air space (see fig. 6). At the beginning, the interface is almost straight at the free surface (fig. 6a) and the vapor gradient is uniform. As the main meniscus progresses the air is saturated with water vapor for $x>1.5-2 \mathrm{~mm}$, the liquid water is at equilibrium with the air (fig. 6c). During the progression of the main meniscus, the computed drying rate $J$ remains constant as the measured drying rate (see fig. 5). The field of vapor density $n$ inside the channel shows that the air close to the main meniscus is saturated with water vapor $\left(n>0.99 \cdot n_{0}\right)$ as soon as the shape of the air/water interface is established (see fig. $6 \mathrm{~b}$ and c). Therefore the water inside the channel is almost at equilibrium with the air and extremely slowly evaporates. Close to the entrance, the gradient of vapor density is high resulting in a high local evaporation flux. This is confirmed by the computation of the local evaporation $F$; its profile is constant in time and decreases exponentially in the channel with a characteristic length $x_{0} \approx 0.4 \mathrm{~mm}$, as shown in fig. 8. The main contribution to the drying rate thus comes from the interface close to the free surface which sets the drying rate; as the shape of the water layer is constant during the CRP, so is the drying rate. The localization of evaporation flux at the channels entrance is similar to the observation in drops where water evaporates mostly at the

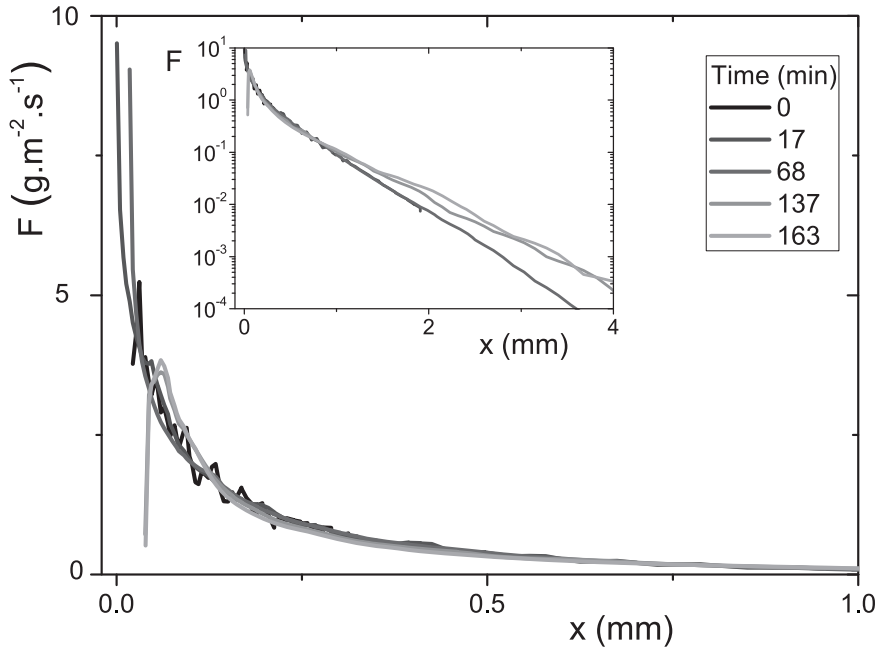

Fig. 8. Evaporating flux $F$ along the air/water interface in the entrance region for five of the configurations shown in fig. 3 .

contact line [29]. Moreover it creates a screening effect in our confined geometry inhibiting evaporation from inside air/water interface. 
As long as the liquid film along the channel sides is continuous we should have an immediate supply of water towards the free surface, leading a priori to a constant drying rate imposed by external conditions down to low saturation. Actually the drying rate is constant down to a saturation of $\phi=0.3$, then it significantly decreases while there is still a continuous film along the channel (see fig. 5).

\subsection{Falling rate period}

Once the finger extends to the end of the channel the evaporating fluid is sourced from the remaining regions; the saturation $\phi \approx \frac{2 w}{W}$ is independent of the channel length $L$. The side layers shrink and the air/water interface moves away from the entrance, as shown in fig. 4. Consequently the drying rate diminishes for $\phi=0.3$, as shown by our simulations (see fig. 5). Accordingly this regime is called the falling rate period. We discuss two simplified scenarios for the evolving shape of the two side layers to better understand our observed drying process.

The decrease of the drying rate occurs as capillary effects are still dominant (see eqs. (2) and (3)). Moreover the channel remains wetted up to its entrance until the very end of the observation $(\varphi \approx 0.06)$. Since this situation is similar to that assumed during the CRP in porous media we expect the drying rate to be constant until the very low residual saturation when permeability in the remaining films is too low to pump water to the surface and a drying front progresses. Here we observe a strong decrease of the drying rate in an unexpected configuration. The drying rate decreases whereas the usual conditions for the CRP are still fulfilled, namely capillary effects able to pump the liquid from the porous medium up to the entrance and continuous films covering the solid surfaces throughout the sample.

This paradox is consistent with water diffusion from the air/water interface. In porous media, it has been shown that the drying rate depends on the saturation as long as the diffusion length $\delta$ is smaller than the distance between wet patches [22]; this situation is similar to our experiments where $\delta \ll W$, therefore the shape of the wet patches influences the drying rate. On the contrary, if this condition is not verified, the drying rate remains constant as the surface saturation decreases and the shape of the water patches does not influence the drying rate. At drying times 137 and $163 \mathrm{~min}$, the drying rate computed by FEM $J$ is lower than in the CRP (see fig. 5) in agreement with the experiments. Thus the evaporation close to the entrance drops due to change in the interface shape observed in the inset of fig. 4b. Moreover the flux decay inside the channel is less pronounced, which means that a longer portion of the interface now significantly contributes to the drying rate (see inset of fig. 8). The geometrical parameters responsible for the decrease are not clear. During the FRP, the water layer thickness $w$ decreases, but also the shape of the air/water interface, which is constant during the CRP, curves and steps away from the entrance (see fig. 4b).
The high contribution to evaporation of the interface close to the free surface of the sample also implies that the thickness of the liquid layers beyond the entrance length has no impact on the drying rate. Since our experimental data show a strong decrease of the drying rate in a regime where the film thickness decreases and the air/water interface shape evolves, we consider two simple configurations based on the water layer thickness $w$ and the angle $\alpha$, i.e. the slope of the interface from the entrance (see schematics in fig. 9). These unrealistic shapes do not represent the evolution of observed interfaces, nevertheless we use them to understand the influence of these two parameters on the drying rate independently.

As the film thickness $(w)$ decreases, the local evaporation $F$ in the channel has a peak at the entrance and decays exponentially with a length scaling with the air layer $r_{\mathrm{c}}=W / 2-w$ (see fig. 9a) which is consistent with 1D diffusion model [11]. The drying rate $J$ decreases linearly with $w$ and the saturation, taken as $\phi=\frac{2 w}{W} ; J$ reaches a minimum value $J_{1} \approx 0.2 \cdot J_{0}$ as $w \rightarrow 0$ (see fig. 9c). The variation of $w$ is not sufficient to explain the observed decrease of the drying rate in the FRP. Keeping $w$ constant, the variation of the slope $\alpha$ has a dramatic impact on the drying rate. $J$ is divided by 2 as $\alpha$ decreases from $90^{\circ}$ to $70^{\circ}$ corresponding to a small decrease of the mean water saturation (see fig. 9c). Thus the change of angle $\alpha$ and variation of the interface at the entrance may explain the decrease of drying rate in a short range of saturation. As $\alpha \rightarrow 0^{\circ}, J$ reaches the same value $J_{1}$ as for decreasing $w$. More generally, $J-J_{1}$ is proportional to $w$. For different $w$, evolution of $\left(J-J_{1}\right) / w$ with $\alpha$ collapses with $\operatorname{cotan}(\alpha) \cdot w$, the distance of junction between the slope and the constant thickness region (see fig. 9b). The decrease of the drying rate extends to a distance $\operatorname{cotan}(\alpha) \cdot w \approx W$. Thus as the entrance region extends over a distance larger than the width of the tube, the drying rate is constant at $J_{1}$ independently of $w$ and $\alpha$.

As $w<H$ eq. (3) underestimates the viscous dissipation which now depends on the corners sharpness [13, $11,27,14]$. The progression of a drying front seems to describe well the drying kinetics until the very low saturation $\phi \approx 10^{-4}$ which is the accuracy limit of our mass measurements. This decrease of the drying rate occurs only at extremely low saturation and after a first relatively long period of falling rate, therefore it is not significant in the drying kinetics of our geometry.

\section{Conclusion}

By combining direct images, mass measurements and FEM analysis we offer an alternative picture for the evaporation process in narrow glass channels. The drying dynamics are set by the shape of the air/water interface and is localised close to the free surface until the last stage where the channel is covered by a very thin film. Accordingly slight changes of the entrance geometry and water distribution at the entrance significantly modify the drying rate. This is contrary to the classical picture that 
(a)

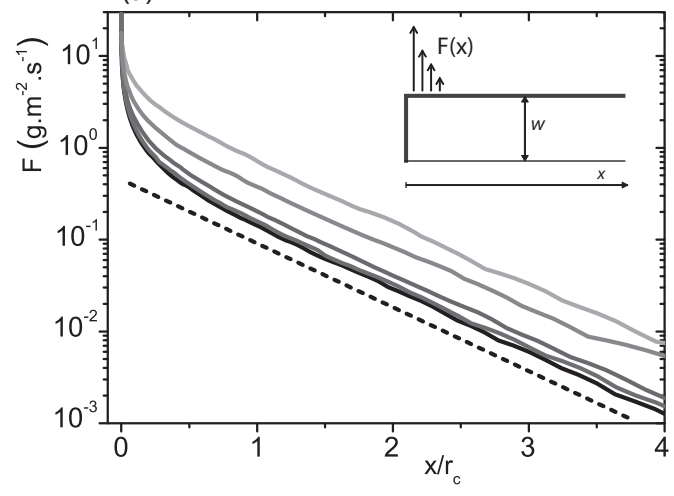

(b)

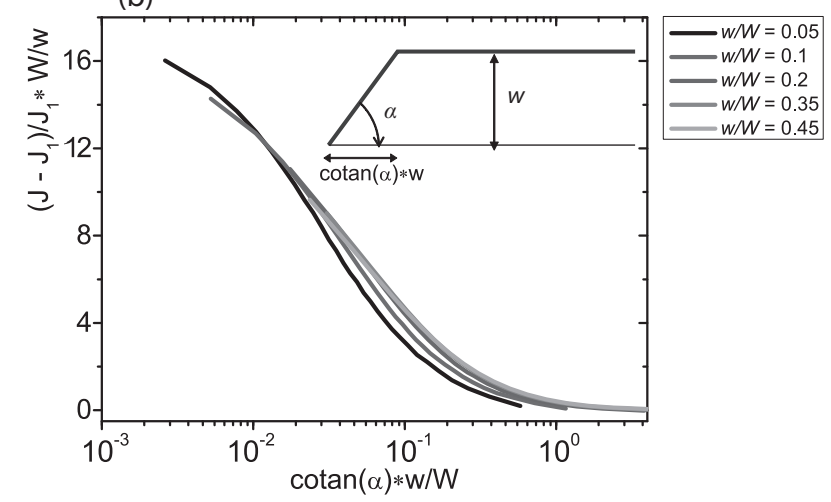

(c)

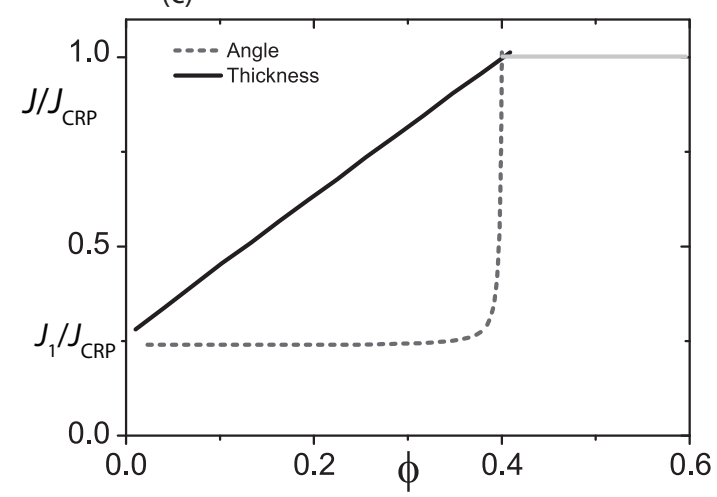

Fig. 9. Simulations of evaporation. (a) Evaporating flux $F$ along the air/water interface parallel to the axis in the configuration where $\alpha=90^{\circ}$. The dotted line emphasizes the exponential decay. (b) Dependence of the drying rate $J$ on the simplified interfacial shape, where the side layer entrance angle $\alpha$ changes, as shown in the inset. By normalizing the drying rate using $J_{1}$, the rate for thin films, and the aspect ratio, $w / W$, five curves corresponding to different $w / W$ collapse. (c) Dependence of the drying rate on the saturation for the two scenarios, where the drying rate is normalized by $J_{\mathrm{CRP}}$ which is the rate during the CRP. The solid curve represents decreasing side layer thickness, $w$, and the dashed curve represents the decreasing entrance angle $\alpha$ of the side layer.

viscous losses are sufficiently large to cause evaporation to proceed via diffusion from a penetrating drying front.

The existence of a thick film is due to capillary equilibrium in our geometry and a similar water distribution may occur in cracks, wedge and microfluidics channels with a spacing of high aspect ratio. Indeed in this case, a small variation of spacing is enough to obtain two thick water layers in the channel edges instead of thin films in the four corners of the section. Water remains at the channel entrance; the viscous pressure drop needs to be small compared to the capillary pressure to provide water to the free surface fast enough regarding the external demand. The same process takes place in a real porous media. At the free surface the evaporative flux $\psi$ is constant in the CRP. Capillarity drives the flow of water to the surface where evaporation occurs and scales with the Laplace pressure $p_{\mathrm{c}}=\gamma / a$, where $a$ is the typical pore size. Viscous dissipation can be written as $\Delta p_{\text {visc }}=\mu \psi L / k$ (with $k$ the permeability)and as long as $\Delta p_{\text {visc }} \ll p_{\text {c }}$, water flow to the surface to compensate evaporation. In complex porous structures, films are usually thick enough to allow water flow $[15,16]$ and to transport elements towards the free surface $[17,18]$. As water content decreases, films become thinner and viscous dissipation increases. When viscous dissipation is too high $\gamma / a<\mu \psi L / k$, the water evaporating close to the free surface is not replaced and a drying front invades the porous medium. Hence the evaporation rate diminishes because water needs to diffuse from drying front to the free surface. In our geometry viscous dissipation is always small compared to capillary pressure and we demonstrate that the Falling Rate Period does not require a receding front. The general process of FRP is not invalidated by our results but we show a mechanism that may apply to some configurations of porous media where it is possible to decrease the drying rate as capillarity is still dominant. Some channel characteristics can be converted to those used for porous media like the permeability or the capillary pressure; but geometrical parameters (aspect ratio) are not clearly defined in a porous structure. Two parameters can be identified: the wettability of the solid for the shape of the air/water interface and the ratio of the diffusion length to the pore size for the drying conditions.

The wetting angle is an important parameter of drying in a porous media, it modifies the amplitude of the capillary forces and thus of the pumping mechanism providing water to the free surface. Moreover the contact angle sets the water distribution at capillary equilibrium as 
the porous medium desaturates, high contact angle limits water preference for corners and small pores which may reduce the drying rate by increasing diffusion length. The pore size also impacts the capillary forces. Moreover the drying rate may decrease with the wetted fraction of the free surface. These results suggest that, as capillary effects are dominant and wet patches are sparse in complex porous structure, the evolution of the exact shape of the liquid/air interface will govern the evaporation rate. We thus conjecture that in certain cases the variations in pore shape of a porous medium close to the free surface can strongly influence drying rate. This insight may help design porous structures with desired evaporation rates.

\section{Author contribution statement}

EK carried out the experiments. EK, SAK, PC developed the models. All authors designed the experiments, discussed the results and wrote the manuscript.

We thank T. Kodger for experimental assistance and helpful discussions; A. Vian for the SEM images.

\section{References}

1. M. Zimmermann, H. Schmid, P. Hunziker, E. Delamarche, Lab Chip 5, 1355 (2005).

2. V. Sartre, M.C. Zaghdoudi, M. Lallemand, Int. J. Therm. Sci. 39, 498 (2000).

3. G.M. Walker, D.J. Beebe, Lab Chip 2, 57 (2002).

4. G. Gauthier, V. Lazarus, L. Pauchard, Langmuir 23, 4715 (2007).

5. S.M. Yang, H. Miguez, G.A. Ozin, Adv. Funct. Mater. 12 , 425 (2002).

6. M. Prat, Int. J. Multiphase Flow 19, 691 (1993).
7. I.N. Tsimpanogiannis, Y.C. Yortsos, S. Poulou, N. Kanellopoulos, A.K. Stubos, Phys. Rev. E 59, 4353 (1999).

8. T.M. Shaw, Phys. Rev. Lett. 59, 1671 (1987).

9. J. Stefan, Sitzungsber. Akad. Wiss. Wien 63, 63 (1871).

10. M. Prat, Int. J. Heat Mass Transf. 50, 1455 (2007).

11. B. Camassel, N. Sghaier, M. Prat, S. Ben Nasrallah, Chem. Engin. Sci. 60, 815 (2005).

12. J.C.T. Eijkel, H.W. Reemeijer, D.C. Hermes, J.G. Bomer, A. Van der Berg, Phys. Rev. Lett. ???, 256107 (2005).

13. F. Chauvet, P. Duru, S. Geoffroy, M. Prat, Phys. Rev. Lett. 103, 1 (2009).

14. H. Wong, S. Morris, C.J. Radke, J. Colloid lntreface Sci. 148, 237 (1992).

15. A. Yiotis, D. Salin, E. Tajer, Y. Yortsos, Phys. Rev. E 85 , 046308 (2012)

16. A. Yiotis, D. Salin, E. Tajer, Y. Yortsos, Phys. Rev. E 86 026310 (2012).

17. L. Pel, A. Sawdy, V. Voronina, J. Cultural Heritage 11, 59 (2010).

18. E. Keita, P. Faure, S. Rodts, P. Coussot, Phys. Rev. E 87, 062303 (2013).

19. P. Faure, P. Coussot, Phys. Rev. E 82, 1 (2010).

20. N. Shahidzadeh-Bonn, A. Azouni, P. Coussot, J. Phys.: Condens. Matter 19, 112101 (2007).

21. N. Shokri, P. Lehmann, D. Or, Water Res. Res. 45, W02415 (2009)

22. M. Suzuki, S. Maeda, J. Chem. Engin. Jpn 1, 26 (1968).

23. P. Coussot, Eur. Phys. J. B 15, 557 (2000).

24. N. Shokri, D. Or, Water Res. Res. 47, 1 (2011).

25. J. Li, B. Cabane, M. Sztucki, L. Goehring, Langmuir 28 , 200 (2012).

26. I. Lesov, S. Tcholokova, N. Denkov, RSC Adv. 4, 811 (2014).

27. M. Dong, I. Chatzis, J. Colloid Interface Sci. 172, 278 (1995).

28. S. Geoffroy, F. Plouraboué, M. Prat, O. Amyot, J. Colloid Interface Sci. 294, 165 (2006).

29. R.D. Deegan, O. Bakajin, T.F. Dupont, G. Huber, S.R. Nagel, T.A. Witten, Nature 389, 827 (1997). 